\title{
Adenomyoepithelioma of the Breast: Which Mimicking Malignancy on Ultrasound Elastography
}

\author{
Shin Young Kim¹, Sun Huh' ${ }^{1}$, Hye Rim Park², Mee Hye $\mathrm{Oh}^{3}$ \\ 'Department of Radiology, Soonchunhyang University Cheonan Hospital, Cheonan; ${ }^{2}$ Department of Radiology, Samsung Medical Center, Seoul; ${ }^{3}$ Department \\ of Pathology, Soonchunhyang University Cheonan Hospital, Cheonan, Korea
}

\begin{abstract}
Adenomyoepithelioma (AME) is a rare breast lesion with balanced proliferation of both epithelial and myoepithelial cells. Patients usually present with a solitary mass without pain and nipple discharge. Although the tumor is generally considered benign, it has potential for local recurrence, metastasis, or malignant change. Approximately 150 cases have been reported in the medical literature, of which 40 cases were malignant or potentially malignant. But among them, elastographic findings have been reported in only two cases and still remain unclear. Herein, we now present a case of breast AME with emphasis on elastography.
\end{abstract}

Keywords: Breast neoplasms; Adenomyoepithelioma; Elasticity imaging techniques

\section{INTRODUCTION}

Adenomyoepithelioma (AME) is an unusual tumor of the breast that is characterized by dual proliferation of epithelial and myoepithelial cells. It was first described by Hamperl [1] in 1970s. Although most AMEs are considered to have a benign clinical course, regional recurrence, distant metastasis, and malignant transformation are also reported. The current treatment of choice is complete excision with sufficient safe margin [2]. It is usually located in the periphery of the breast [3], but is also found centrally or around the areola [2]. Several case reports and studies on mammography and ultrasonography (USG) findings of AME are reported; however, reports of ultrasound (US) elastography for evaluation of AME are rare [4]. Hence, we described the radiologic finding of breast AME with emphasis on US elastography.

\section{CASE REPORT}

A 45-year-old female was referred to Soonchunhyang University Cheonan Hospital for a slowly growing left breast mass of 1-year duration that was confirmed by USG in a local clinic. Clinically, the patient had no complaints of pain or other symptoms such as nipple discharge. On physical examination, there was no palpable mass in breast, and no evidence of associated axillary lymphadenopathy.

Mammography revealed an approximately $0.5-\mathrm{cm}$ sized oval circumscribed equal density nodule in lower outer quadrant (LOQ) of left breast in craniocaudal view, but it was not definitely delineated in mediolateral oblique view (Fig. 1). Breast USG revealed an approximately $0.7-\mathrm{cm}$ sized circumscribed oval hypoechoic nodule in LOQ. On color Doppler, mild internal vascularity was apparent within the mass (Fig. 2). Sonographic finding was indicative of a growing benign lesion rather than malignancy. So we classified the tumor as BI-RADS 4A based on the Breast Imaging and Reporting Data System. In addition, US elastography was performed to evaluate the lesion stiffness.

Unlike USG, elastography revealed red color mapping on breast lesion, which is suggestive of malignancy (Fig. 3) [5]. In evaluation with Tsukuba score, the lesion was stiffer than the surrounding tissue and was the same size on the elastogram and B-mode US, so it was thought to be score 4 [6]. Patient was worried about the breast mass and wanted surgical removal. We performed core biopsy and pathologic report was suggestive of AME.

Thereafter, US-guided localized excisional biopsy was per-
Correspondence to: Shin Young Kim

Department of Radiology, Soonchunhyang University Cheonan Hospital, 31 Suncheonhyang 6-gil, Dongnam-gu, Cheonan 31151, Korea

Tel: +82-41-570-3515, Fax: +82-41-579-9026, E-mail: c87093@schmc.ac.kr

Received: Mar. 26, 2019 / Accepted after revision: May 22, 2019
(C) 2019 Soonchunhyang Medical Research Institute This is an Open Access article distributed under the terms of the Creative Commons Attribution Non-Commercial License (http://creativecommons.org/licenses/by-nc/4.0/). 
formed for left breast mass (Fig. 4). Histologically, the mass consisted of small glands, i.e., epithelial cells surrounded by myoepithelial cell layers (Fig. 5). Also, immunohistochemical staining was positive for p63, CK5/6, and smooth muscle myosin heavy chain (Fig. 6).

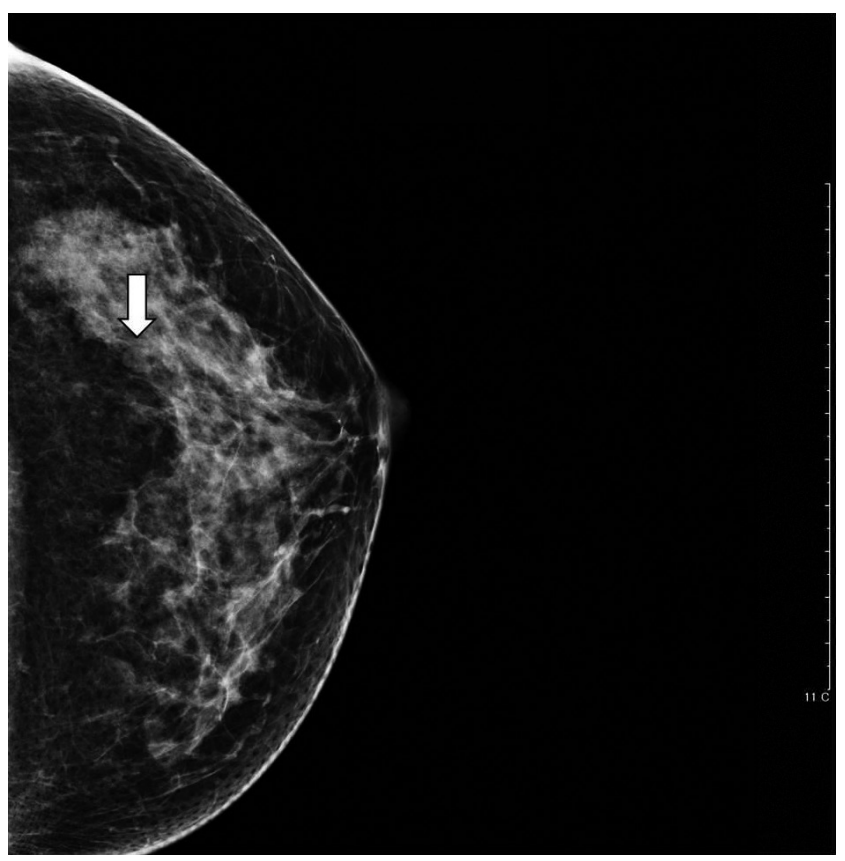

Fig. 1. In craniocaudal view, mammography revealed 0.5-cm sized oval circumscribed equal density nodule (arrow) without calcification or spiculation in left breast.

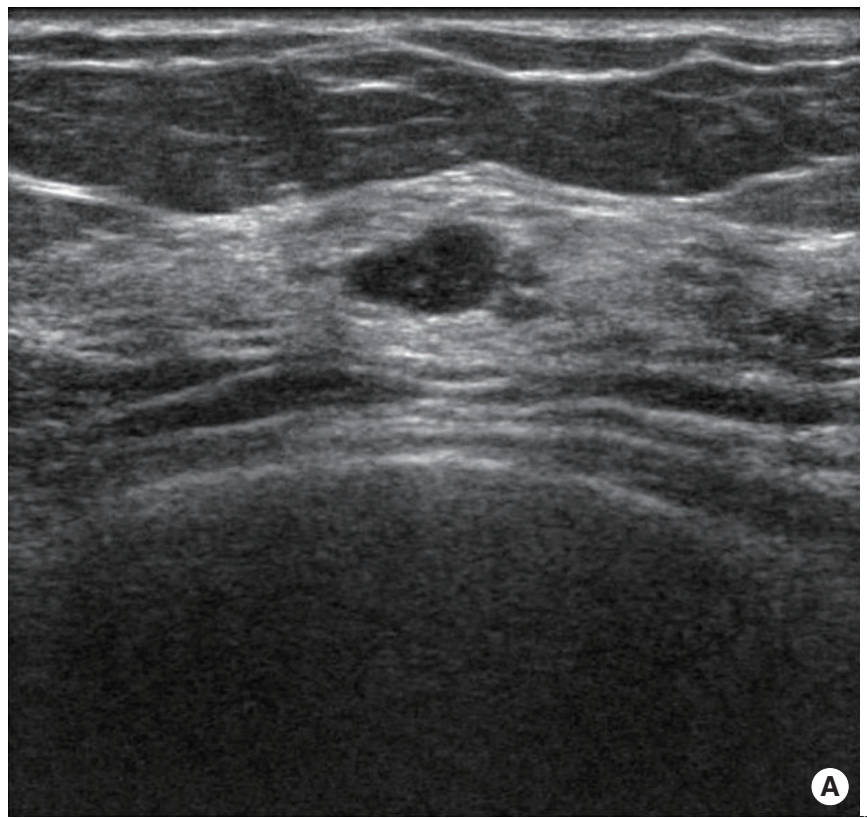

Finally, the pathologic result of the resected nodule confirmed AME. No additional postoperative treatment was done and the patient showed no evidence of recurrence during the 2-month follow-up period. And written informed consent was obtained from the patient.

\section{DISCUSSION}

Myoepithelial cells are a normal component of the mammary duct system, located between the epithelial layer and basement membrane of ducts and acini. Hyperplasia of myoepithelial cells can be found in many benign lesions such as sclerosing adenosis and papilloma [4]. Tavassoli [7] classified breast myoepithelial lesions into three categories including (1) myoepitheliosis (2) AME, and (3) myoepithelial carcinoma. Additionally, AMEs are subdivided into tubular, lobulated, or spindle subtypes.

So far, most cases have been reported in females with rare cases in males [8]. Benign AME reportedly shows nonspecific radiologic findings [9]. In a review of the literature, mammography shows no characteristic findings in cases of AME.

Yoon and Chitale [8] described AME as a round or lobulated, dense, mostly circumscribed mass, occasionally with partial indistinct margins. In addition, Adejolu et al. [2] reported an irregular noncalcified mass with microlobulated margins as the predominant mammographic findings in AME. In our study, the pa-

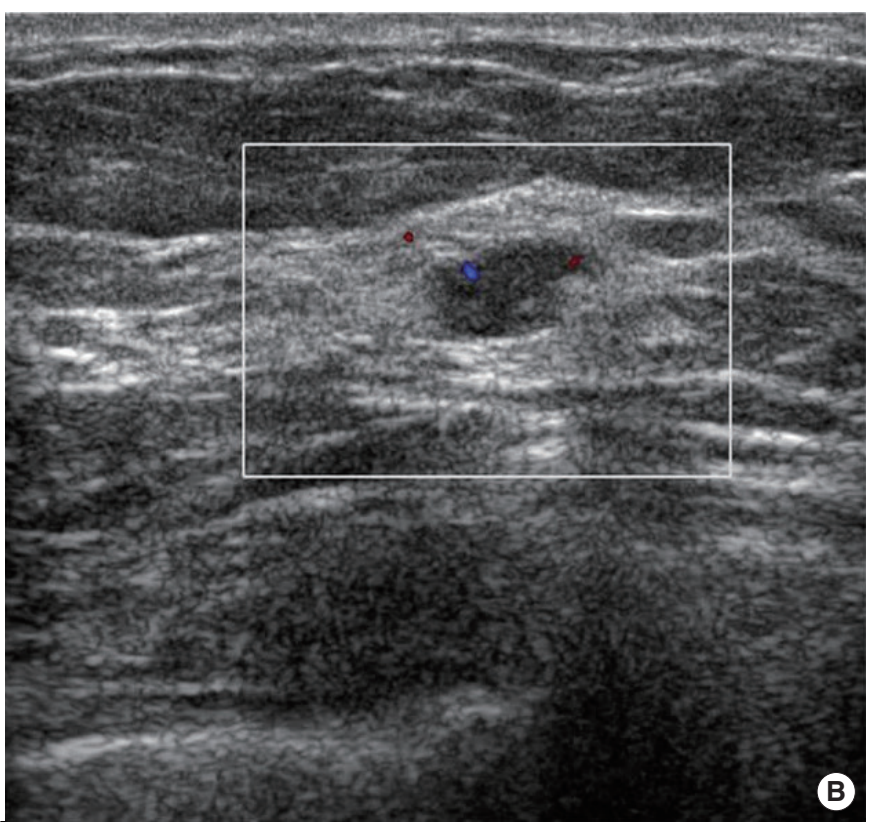

Fig. 2. A 0.7-cm sized circumscribed oval hypoechoic nodule in the left breast on ultrasonography (A) and color-Doppler scan (B) demonstrated mild internal vascularity. 

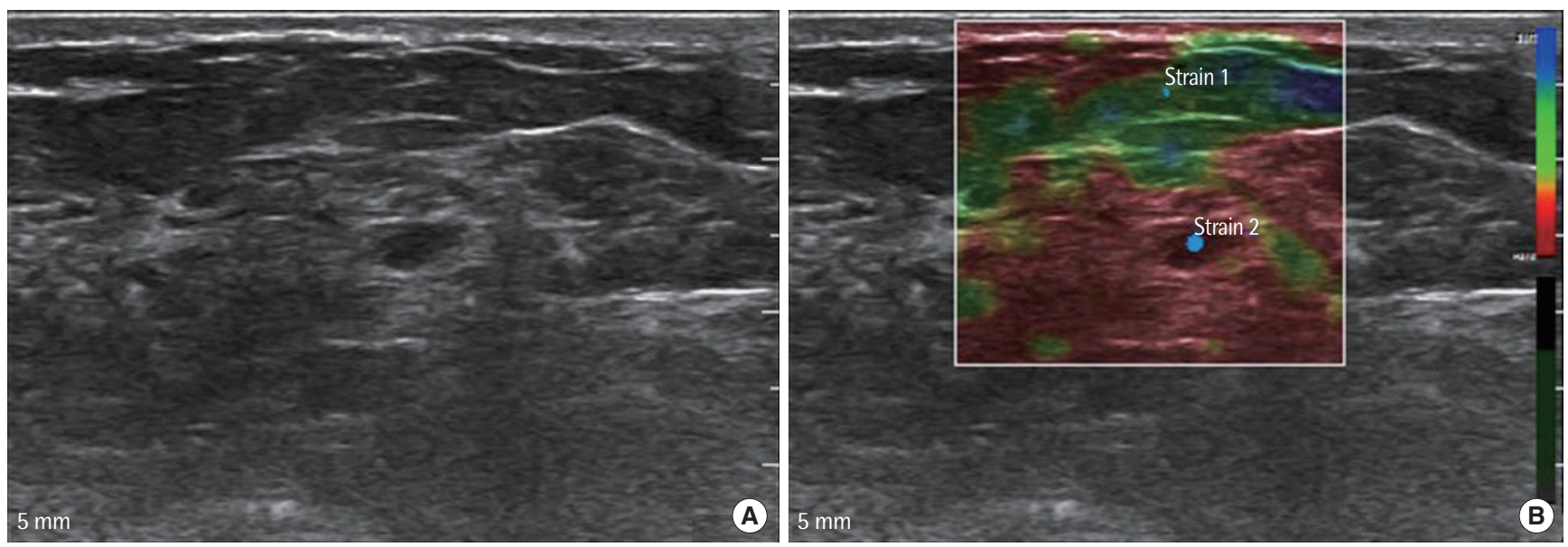

Fig. 3. (A, B) Quantitative elastography of the lesion revealed red component of the mass, suggestive of high strain lesion.

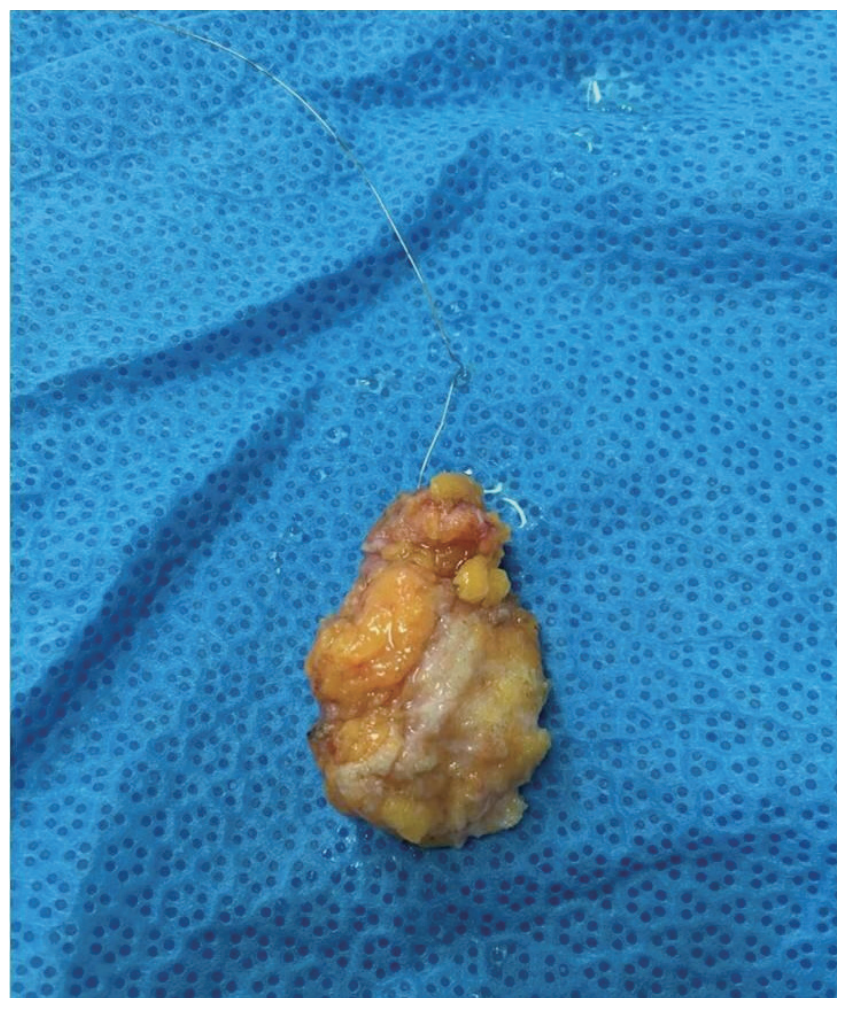

Fig. 4. Needle localized excision and biopsy specimen shows well circumscribed mass with fat tissue.

tient showed a round and circumscribed mass lesion with no definite microcalcification.

The most common sonographic findings are hypoechogenicity, increased peripheral vascularity [9]. Adejolu et al. [2] described USG findings of AME as solid, hypoechoic mass with microlobulated margins with possible posterior acoustic enhancement and associated hypervascularity. Gkali et al. [4] reported AME of mixed pattern with solid isoechoic and cystic part and internal vascularity. Likewise, our case showed hypoechoic lesion with internal vascularity.

Sonographic elastography is a new imaging technique that enables evaluation of the breast lesion stiffness [5]. The two most commonly used elastographic techniques in the breast are strain elastography and shear wave (SW) elastography. In strain elastography, stress is applied by repeated manual compression of the transducer, and the amount of lesion deformation relative to the surrounding normal tissue is measured and displayed in color.

Thus, significant interobserver variability has been found. On the other hand, SW elastography allows measurement of the propagation speed of SWs within the tissue to locally quantify its stiffness in kilopascals or meters per second using acoustic radiation force impulse. So, it has an advantage of reproducibility [10]. Benign nodules are deformable, whereas malignant lesions tend to be hard and less elastic [5]. Currently, very few reports and studies on evaluation of AME with elastography are published in the literature.

The elastogram, which reflects the relative elasticity of the tissues, is created as a color coded map (the areas of great stiffness are coded in red, those which are more deformable in blue, and green indicates intermediate levels of elasticity). Lighter shades of the base color reflect the different degrees of tissue deformability and correlate with the dynamic range of the analytical system [5]. Like the previous report, our patient showed the entire lesion as red, indicating a stiff lesion, suggestive of malignancy. Considering the only elastographic findings, it is suggestive of malignancy with stiffness. 
Kim SY, et al. • Extremely Rare Adenomyoepithelioma of the Breast
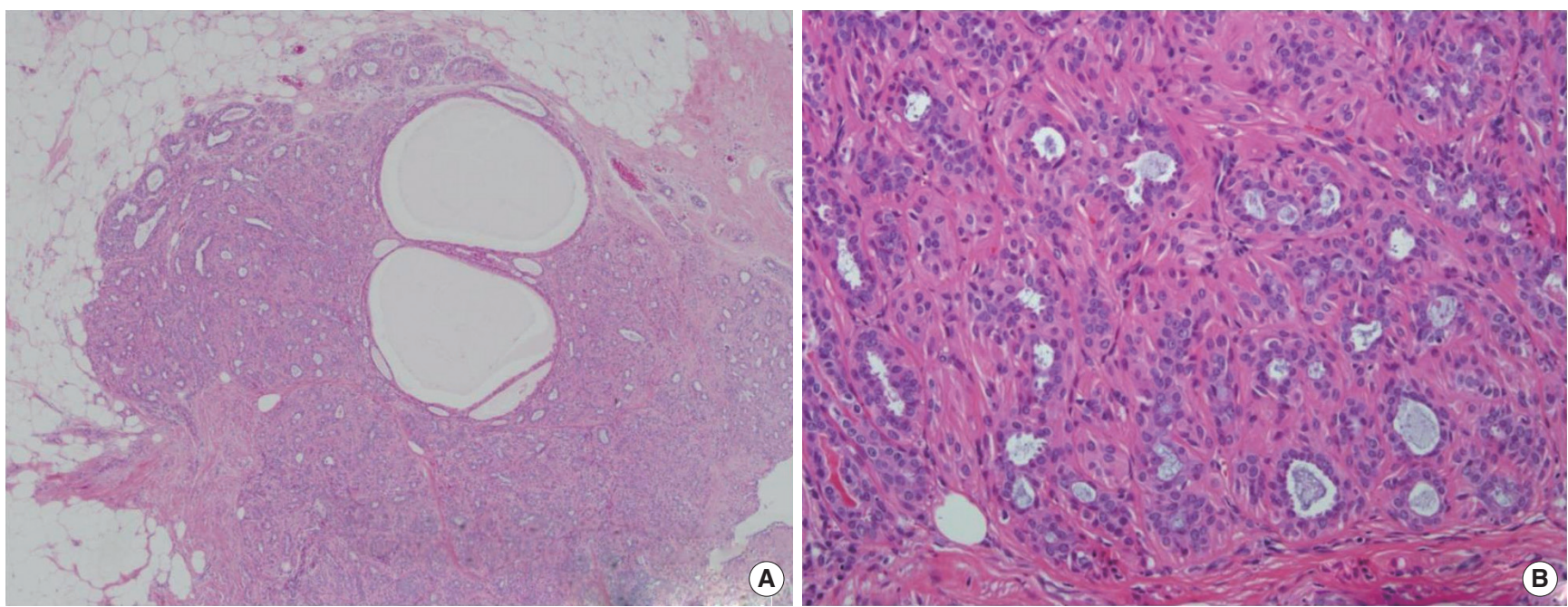

Fig. 5. (A) A relatively well circumscribed tumor composed of myoepithelial cell and epithelial cells showing focal cystic change with apocrine differentiation (H\&E, $\times 40)$. (B) Small glands composed of epithelial cells surrounded by spindle or polygonal shaped myoepithelial cells (H\&E, $\times 200)$.
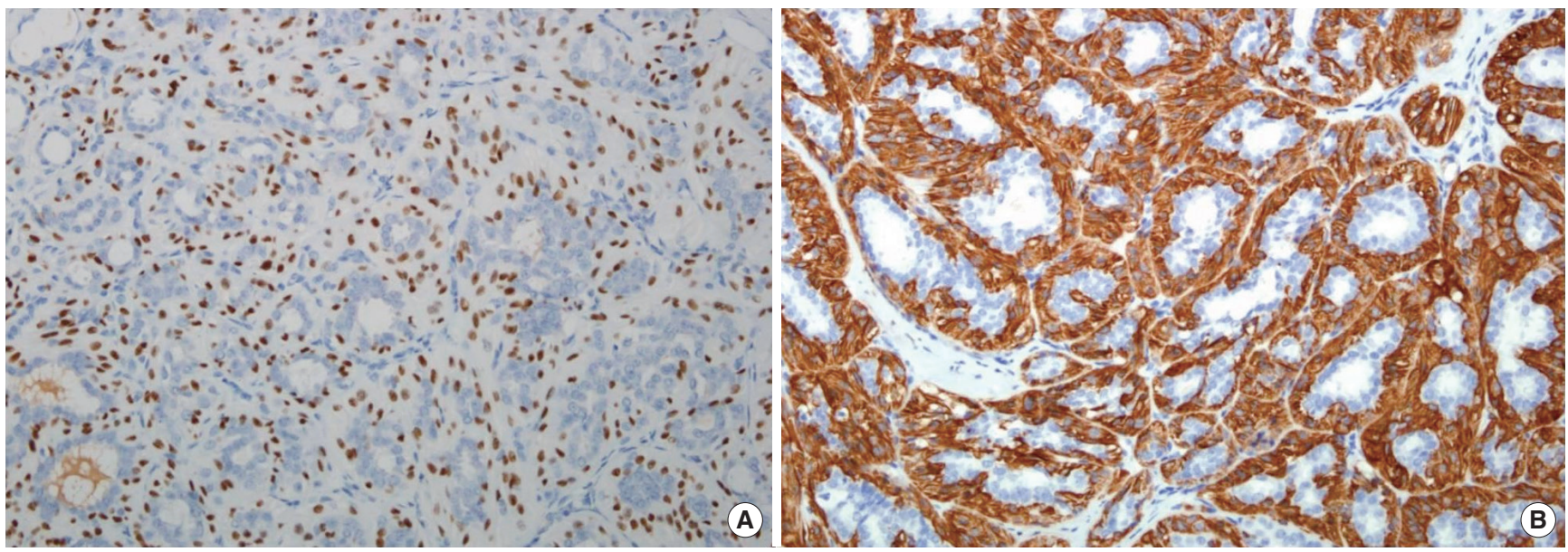

Fig. 6. (A, B) Immunostains for smooth muscle myosin heavy chain and p63 revealed diffuse positive reaction in myoepithelial cells $(\times 200)$.

When classified according to the Tsukuba score, the lesion was stiffer than surrounding lesion and considered to be the same size when compared with the conventional US.

In the literature review, some case reports describe magnetic resonance imaging (MRI) findings for AME. Lee et al. [9] reported a patient who underwent MRI and positron emission tomography-computed tomography (PET-CT). On MRI, the patient showed an oval, smooth mass with homogenous enhancement and a delayed persistent pattern on the kinetic curve. No fluorodeoxyglucose uptake was noted on PET-CT [9].

Differential diagnosis of AME includes papilloma with prominent myoepithelial cells, tubular adenoma, sclerosing adenosis, fibroadenoma, and pleomorphic adenoma [4]. Most AMEs are con- sidered as benign lesion with a very low malignant potential. However, regional recurrence, distant metastasis, and malignant transformation have also been reported [2].

Malignant transformation in this lesion is rare and can derive from epithelial and myoepithelial cells; one or both components can show malignant change [8]. The prognosis of patients with benign AME of the breast is considered good when the initial treatment is adequate [4]. Due to the potential for malignant change and local recurrence, a total resection with sufficiently wide margin with uninvolved breast tissue is required in initial surgical treatment $[3,7,8]$.

In conclusion, $\mathrm{AME}$ of breast is a rare neoplasm with a mostly benign clinical course. The ideal treatment of these tumors is 
complete excision with wide safe margins due to potential for local recurrence and malignant transformation. Patients require close and regular follow-up postoperative. The elastographic finding from our study could help in the diagnosis and evaluation of AME.

\section{REFERENCES}

1. Hamperl H. The myothelia (myoepithelial cells): normal state; regressive changes; hyperplasia; tumors. Curr Top Pathol 1970;53:161-220.

2. Adejolu M, Wu Y, Santiago L, Yang WT. Adenomyoepithelial tumors of the breast: imaging findings with histopathologic correlation. AJR Am J Roentgenol 2011;197:W184-90.

3. Rosen PP. Adenomyoepithelioma of the breast. Hum Pathol 1987;18: 1232-7.

4. Gkali CA, Chalazonitis AN, Feida E, Dimitrakakis C, Sotiropoulou M.
Breast adenomyoepithelioma: ultrasonography, elastography, digital mammography, contrast-enhanced digital mammography, and pathology findings of this rare type of breast tumor. Ultrasound Q 2015;31:185-8.

5. Goddi A, Bonardi M, Alessi S. Breast elastography: a literature review. J Ultrasound 2012;15:192-8.

6. Balleyguier C, Ciolovan L, Ammari S, Canale S, Sethom S, Al Rouhbane $\mathrm{R}$, et al. Breast elastography: the technical process and its applications. Diagn Interv Imaging 2013;94:503-13.

7. Tavassoli FA. Myoepithelial lesions of the breast: myoepitheliosis, adenomyoepithelioma, and myoepithelial carcinoma. Am J Surg Pathol 1991; 15:554-68.

8. Yoon JY, Chitale D. Adenomyoepithelioma of the breast: a brief diagnostic review. Arch Pathol Lab Med 2013;137:725-9.

9. Lee JH, Kim SH, Kang BJ, Lee AW, Song BJ. Ultrasonographic features of benign adenomyoepithelioma of the breast. Korean J Radiol 2010;11:522-7.

10. Chang JM, Won JK, Lee KB, Park IA, Yi A, Moon WK. Comparison of shear-wave and strain ultrasound elastography in the differentiation of benign and malignant breast lesions. AJR Am J Roentgenol 2013;201: W347-56. 